\title{
Frame-based and robot-assisted insular stereo-electroencephalography via an anterior or posterior oblique approach
}

\author{
Kathrin Machetanz, MD, ${ }^{1,2}$ Florian Grimm, MD, ${ }^{1,2}$ Thomas V. Wuttke, MD, ${ }^{1,3}$ Josua Kegele, MD, ${ }^{3}$ \\ Holger Lerche, MD, ${ }^{3}$ Marcos Tatagiba, MD, ${ }^{1}$ Sabine Rona, MD, PhD, ${ }^{1}$ Alireza Gharabaghi, MD, ${ }^{1,2}$ \\ Jürgen Honegger, MD, ${ }^{1}$ and Georgios Naros, MD ${ }^{1,2}$
}

\begin{abstract}
${ }^{1}$ Department of Neurosurgery; ${ }^{2}$ Division of Functional and Restorative Neurosurgery, Department of Neurosurgery; and ${ }^{3}$ Department of Epileptology, Eberhardt Karls University, Tuebingen, Germany
\end{abstract}

\begin{abstract}
OBJECTIVE There is an increasing interest in stereo-electroencephalography (SEEG) for invasive evaluation of insular epilepsy. The implantation of insular SEEG electrodes, however, is still challenging due to the anatomical location and complex functional segmentation in both an anteroposterior and ventrodorsal (i.e., superoinferior) direction. While the orthogonal approach $(\mathrm{OA})$ is the shortest trajectory to the insula, it might insufficiently cover these networks. In contrast, the anterior approach (AOA) or posterior oblique approach (POA) has the potential for full insular coverage, with fewer electrodes bearing a risk of being more inaccurate due to the longer trajectory. Here, the authors evaluated the implantation accuracy and the detection of epilepsy-related SEEG activity with AOA and POA insular trajectories.

METHODS This retrospective study evaluated the accuracy of 220 SEEG electrodes in 27 patients. Twelve patients underwent a stereotactic frame-based procedure (frame group), and 15 patients underwent a frameless robot-assisted surgery (robot group). In total, 55 insular electrodes were implanted using the AOA or POA considering the insular anteroposterior and ventrodorsal functional organization. The entry point error (EPE) and target point error (TPE) were related to the implantation technique (frame vs robot), the length of the trajectory, and the location of the target (insular vs noninsular). Finally, the spatial distribution of epilepsy-related SEEG activity within the insula is described.

RESULTS There were no significant differences in EPE (mean $0.9 \pm 0.6$ for the nonsinsular electrodes and $1.1 \pm 0.7$ $\mathrm{mm}$ for the insular electrodes) and TPE (1.5 \pm 0.8 and $1.6 \pm 0.9 \mathrm{~mm}$, respectively), although the length of trajectories differed significantly $(34.1 \pm 10.9$ and $70.1 \pm 9.0 \mathrm{~mm}$, repsectively). There was a significantly larger EPE in the frame group than in the robot group $(1.5 \pm 0.6$ vs $0.7 \pm 0.5 \mathrm{~mm})$. However, there was no group difference in the TPE $(1.5 \pm 0.8$ vs 1.6 $\pm 0.8 \mathrm{~mm})$. Epilepsy-related SEEG activity was detected in $42 \%(23 / 55)$ of the insular electrodes. Spatial distribution of this activity showed a clustering in both anteroposterior and ventrodorsal directions. In purely insular onset cases, subsequent insular lesionectomy resulted in a good seizure outcome.

CONCLUSIONS The implantation of insular electrodes via the AOA or POA is safe and efficient for SEEG implantation covering both anteroposterior and ventrodorsal functional organization with few electrodes. In this series, there was no decrease in accuracy due to the longer trajectory of insular SEEG electrodes in comparison with noninsular SEEG electrodes. The results of frame-based and robot-assisted implantations were comparable.
\end{abstract}

https://thejns.org/doi/abs/10.3171/2020.10.JNS201843

KEYWORDS frameless stereotactic surgery; insular epilepsy; accuracy; robot-assisted surgery; stereo-electroencephalography

$\mathrm{T}$ HE use of stereo-electroencephalography (SEEG) within the insula to determine seizure onset prior to resective surgery is well established for patients with medically intractable epilepsy. ${ }^{1-3}$ However, implantation of SEEG electrodes in the insula is still challenging due to its anatomical features. First, the insular cortex is located deep inside the sylvian fissure, enclosed and covered by the frontoparietal and temporal opercula. Second, the cytoarchitectonic and functional structure of the insula is complex and poorly understood. ${ }^{4,5}$ Despite its name, the

ABBREVIATIONS $A O A=$ anterior oblique approach; $E E G$ = electroencephalography; $E P E=$ entry point error; $F C D=$ focal cortical dysplasia; $F R E=$ fiducial registration error; OA = orthogonal approach; $\mathrm{POA}=$ posterior oblique approach; $\mathrm{SEEG}=$ stereo-EEG; TPE = target point error; VEEG = video-EEG.

SUBMITTED May 16, 2020. ACCEPTED October 21, 2020.

INCLUDE WHEN CITING Published online April 30, 2021; DOI: 10.3171/2020.10.JNS201843. 
insula is not isolated but highly interconnected with other brain regions, ${ }^{4-6}$ resulting in a very heterogeneous seizure semiology. In general, the insula is considered to be organized in an anteroposterior direction as a function of each insular gyrus. ${ }^{4,5}$ However, less attention is paid to the ventrodorsal (i.e., inferosuperior) organization concentrically arranged around the anterobasally situated apex of the insula. ${ }^{4,6}$

While several stereotactic approaches have been described, the orthogonal approach (OA; i.e., the transopercular placement of axially oriented electrodes) is the most common trajectory to the insula. ${ }^{8}$ Although being the shortest path, the OA is hazardous due to the proximity of the sylvian fissure and its vessels. ${ }^{9,10}$ In contrast, the anterior oblique approach (AOA) and posterior oblique approach (POA) from frontal and parietal entry points minimize pial violations; however, these approaches are potentially less accurate due to the longer trajectory. ${ }^{11,12}$ Moreover, the common OA, with 3-4 trajectories and only a few contacts per electrode within the cortex,${ }^{13}$ usually struggles to cover the complete anteroposterior and ventrodorsal extension of the insula. Thus, focally clustered epilepsy-related EEG activity within distinct insular circuits might be missed by orthogonal electrodes. Multiplying the number of OA trajectories, however, might also increase the vascular risk. In contrast, the AOA or POA increases the number of serviceable contacts ${ }^{13,14}$ and with the course of the trajectories has the potential for full insular coverage with fewer electrodes.

Currently, there are two competing surgical techniques for insular SEEG implantation: the classic frame-based stereotaxy and the robot-assisted procedure. Representing the gold standard, frame-based stereotactic implantations have been shown to be safe and precise but very time consuming. ${ }^{15}$ In contrast, robot-assisted SEEG implantation techniques are suggested to overcome this drawback by enabling a faster and easier workflow. However, their accuracy is a matter of debate. ${ }^{16-18}$ This obstacle could gain special importance in long trajectories (e.g., for insular SEEG). Thus, most centers with robotic systems apply the OA..$^{19}$ However, there are only few data available on robotassisted electrode implantations for insular SEEG via an AOA or POA.

The present study aimed to analyze the safety and the accuracy of placing insular electrodes using a frame-based or robot-assisted AOA or POA in comparison with other SEEG targets and to describe a potential spatial clustering of epilepsy-related EEG activity within the insula.

\section{Methods \\ Patient Population}

This retrospective study included 27 consecutive patients (mean age $[ \pm \mathrm{SD}] 25.0 \pm 16.0$ years [range 2.8-57.9 years], 17 female) who underwent implantation of electrodes for SEEG in the neurosurgical department of the University of Tuebingen between 2017 and 2020 by a single neurosurgeon (G.N.). Twelve patients (mean age 23.2 \pm 17.0 years [range 2.8-56.5 years], 9 female) underwent frame-based surgery (frame group) and 15 patients (mean age $26.5 \pm 15.7$ years [range 5.9-57.9 years], 8 female) underwent a robot-assisted surgery (ROSA One, Zimmer Biomet; robot group). The frame-based implantations were performed in the months prior to the installation of the robotic unit in September 2018. Notably, 10 (37\%) of 27 patients were children (age $<18$ years) and were equally distributed between the frame and robot groups. The study was approved by the local ethics committee of the Eberhardt Karls University Tuebingen and performed in accordance with the Declaration of Helsinki.

\section{Clinical Data and Seizure Semiology}

All patients underwent a detailed history taking, highresolution MRI, long-term scalp video-EEG (VEEG) monitoring, and neuropsychological assessments. Preoperative high-resolution MR images were evaluated by several independent reviewers (experienced neuroradiologists, neurologists [H.L. and S.R.], and experienced neurosurgeons [J.H. and G.N.]). SEEG was indicated when the noninvasive studies provided contradicting information or were insufficient to delineate the seizure onset zone. Seizure semiology was reviewed from clinical descriptions by the patients and their families, and the videos recorded during VEEG and SEEG were reviewed by a single experienced reviewer (S.R.) and categorized according to the classification of the International League Against Epilepsy from 2017. ${ }^{20}$ Epilepsy-related EEG activity was evaluated on the VEEG and SEEG recordings by a single experienced reviewer (S.R.). Seizure onset was classified as purely insular when ictal onset was confined to the insular cortex, including the circular sulcus. The results of the noninvasive workup are summarized in Table 1. Seizure outcome of a resective surgery at the latest appointment was assessed according to Engel's classification. ${ }^{21}$

\section{Preoperative Imaging and Trajectory Planning}

In all patients, preoperative MRI, including high-resolution T1-weighted contrast-enhanced sequences (isovoxel $1 \mathrm{~mm}$ ), was performed. Trajectories were planned based on the preoperative MR images using the Brainlab iPlan cranial 3.0 software (Brainlab AG) in the frame group or the planning software of the ROSA in the robot group. An AOA or POA was chosen for the placement of the insular electrodes. We perform an AOA via an anterior frontal entry point at the superior or medial frontal gyrus to target the accessory gyrus as well as the anterior short and middle short insular gyri representing social-emotional and cognitive functional networks, respectively. ${ }^{5,22}$ The AOA with a posterior frontal entry point is used to target the region between the middle and posterior short gyri involved in chemosensory circuits. ${ }^{5,22}$ The sensorimotor networks within the anterior and posterior long gyri posterior to the central insular sulcus ${ }^{5,22}$ are targeted by a POA via an entry point at the postcentral gyrus or superior parietal lobule. In the coronal view, SEEG electrodes were planned tangentially within the insular cortex to cover the ventrodorsal functional circuits as described by Augustine $(1996)^{4,6}$ (Fig. 1).

\section{Surgical Procedure}

Prior to surgery, the stereotactic frame (Radionics 
Brown-Roberts-Wells, BRW; frame group) or 5 bone fiducials (WayPoint, FHC; robot group) were placed under local anesthesia. Subsequently, a $0.75-\mathrm{mm}$ contrast-enhanced CT scan (multislice CT scanner, Siemens Medical $\mathrm{GmbH}$ ) was acquired in all patients and fused to the MR image. All intraoperative procedures were performed under general anesthesia with the patient placed supine. In the robot group, the patient's head was fixed in a Mayfield skull clamp and coupled to the robot. Subsequently, registration was performed based on the 3D CT scan utilizing the 5 bone fiducials. The principles of bone fiducial registration in ROSA-assisted procedures are described in detail elsewhere. ${ }^{18}$ Electrode implantation (Dixi Médical) was performed in an identical standardized manner in both groups. After indication of the entry point by the stereotactic device, the patient's hair was shaved locally. Skin asepsis was followed by a stab incision. The trepanation was performed with a motorized 2.1-mm twist drill (Acculan 4, Aesculap AG) according to the individual bone thickness. In robot-based implantations, the robot arm is moved as close as possible to the head to avoid slipping along the skull, even in very oblique trajectories. The dura and the surface of the cortex were coagulated and perforated using a monopolar coagulation device. The anchor bolt was placed, and a stylet was introduced for electrode guidance. Finally, the electrode was introduced and fixed with the adjacent screw to the anchor bolt. Subsequently, 3 patients underwent an additional implantation of $6.3 \pm 1.5$ [range 6-8] subdural electrodes. These patients were excluded from the analysis regarding implantation accuracy and surgical procedure time. Postoperatively, every patient underwent high-resolution T1-weighted MRI controlling for the final electrode location and intracranial complications. In cases in which the MR image was not available directly after surgery, additional high-resolution CT scanning was performed.

\section{Data Analysis}

The postoperative CT and MRI scans were retrospectively fused with the preoperative planning in the iPlan cranial 3.0 software (Brainlab) or ROSA software. Entry point error (EPE) and target point error (TPE) were defined as the distance of the actual entry point (at the bone surface) or target point (at the level of the lowest electrode contact) to the originally planned trajectory axis. ${ }^{23}$ The EPE was analyzed based on postoperative CT findings, if available (in 16 patients with 130 SEEG implantations). The TPE was analyzed based on postoperative MRI. Patients with concomitant implantation of subdural electrodes $(n=3)$ and those with intracranial complications $(\mathrm{n}=1)$ were removed from the analysis concerning implantation accuracy (with 192 SEEG implantations and 23 patients remaining). Subdural electrodes and craniotomy might affect accuracy analysis by mass effect and brain shift. Furthermore, the length of the electrode (i.e., from dura to the lowest contact) and the localization of the electrode (frontal, temporal, parietal, occipital, or insular) as well as the number of contacts within the insula were noted. For the robot group, the duration and the accuracy of the registration process were noted. The accuracy of the registration process was assessed by the fiducial registra-
TABLE 1. Details of 27 patients undergoing insular SEEG via an AOA or POA

\begin{tabular}{|c|c|}
\hline & Value \\
\hline Mean age, yrs & $25.0 \pm 16.0(2.78-57.9)$ \\
\hline Female/male & $17: 10$ \\
\hline Age at seizure onset, yrs & $11.3 \pm 11.5(0-47)$ \\
\hline Epilepsy duration, yrs & $13.8 \pm 11.2(0.8-42.5)$ \\
\hline \multicolumn{2}{|l|}{ Type of seizure } \\
\hline FIAS & $8(29.6)$ \\
\hline FBTCS & $19(70.4)$ \\
\hline \multicolumn{2}{|l|}{ Symptoms/signs } \\
\hline \multicolumn{2}{|l|}{ Motor } \\
\hline Tonic & $12(44.4)$ \\
\hline Automatisms & $11(40.7)$ \\
\hline Clonus & $2(7.4)$ \\
\hline Hypermotoric & $1(3.7)$ \\
\hline Myoclonic & $1(3.7)$ \\
\hline \multicolumn{2}{|l|}{ Nonmotor } \\
\hline Sensory & $9(33.3)$ \\
\hline Cognitive & $4(14.8)$ \\
\hline Epigastric & $3(11.1)$ \\
\hline Emotional & $3(11.1)$ \\
\hline Visual & $3(11.1)$ \\
\hline Auditory & $1(3.7)$ \\
\hline Autonomic & $1(3.7)$ \\
\hline \multicolumn{2}{|l|}{ VEEG seizure onset } \\
\hline Temporal & $14(51.9)$ \\
\hline Frontal & $12(44.4)$ \\
\hline Central & $10(37.0)$ \\
\hline Parietal & $7(25.9)$ \\
\hline Occipital & $7(25.9)$ \\
\hline Multifocal & $1(3.7)$ \\
\hline \multicolumn{2}{|l|}{ MRI findings } \\
\hline FCD & $13(48.1)$ \\
\hline Nonlesional & $7(25.9)$ \\
\hline HS & $4(14.8)$ \\
\hline Miscellaneous* & $3(11.1)$ \\
\hline Temporal & $7(25.9)$ \\
\hline Insular & $7(25.9)$ \\
\hline Frontal & $5(18.5)$ \\
\hline Parietal & $4(14.8)$ \\
\hline Multifocal & $1(3.7)^{\prime}$ \\
\hline
\end{tabular}

FBTCS = focal to bilateral tonic-clonic seizure; FIAS = focal with impaired awareness seizure; HS = hippocampal sclerosis.

Values represent the number of patients (\%) unless stated otherwise. Mean values are presented as the mean \pm SD (range).

* For example, gliosis.

tion error (FRE): the root mean square distance between the fiducial localization in the image space and the fiducial localization in the physical space. ${ }^{24}$

Finally, standardized time stamps were documented during surgery defining the overall surgical procedure time (i.e., surgeon's entry/exit to/from the operating room). Dividing the surgical procedure time by the number of SEEG trajectories resulted in the implantation time per electrode. Patients with concomitant implantation of sub- 

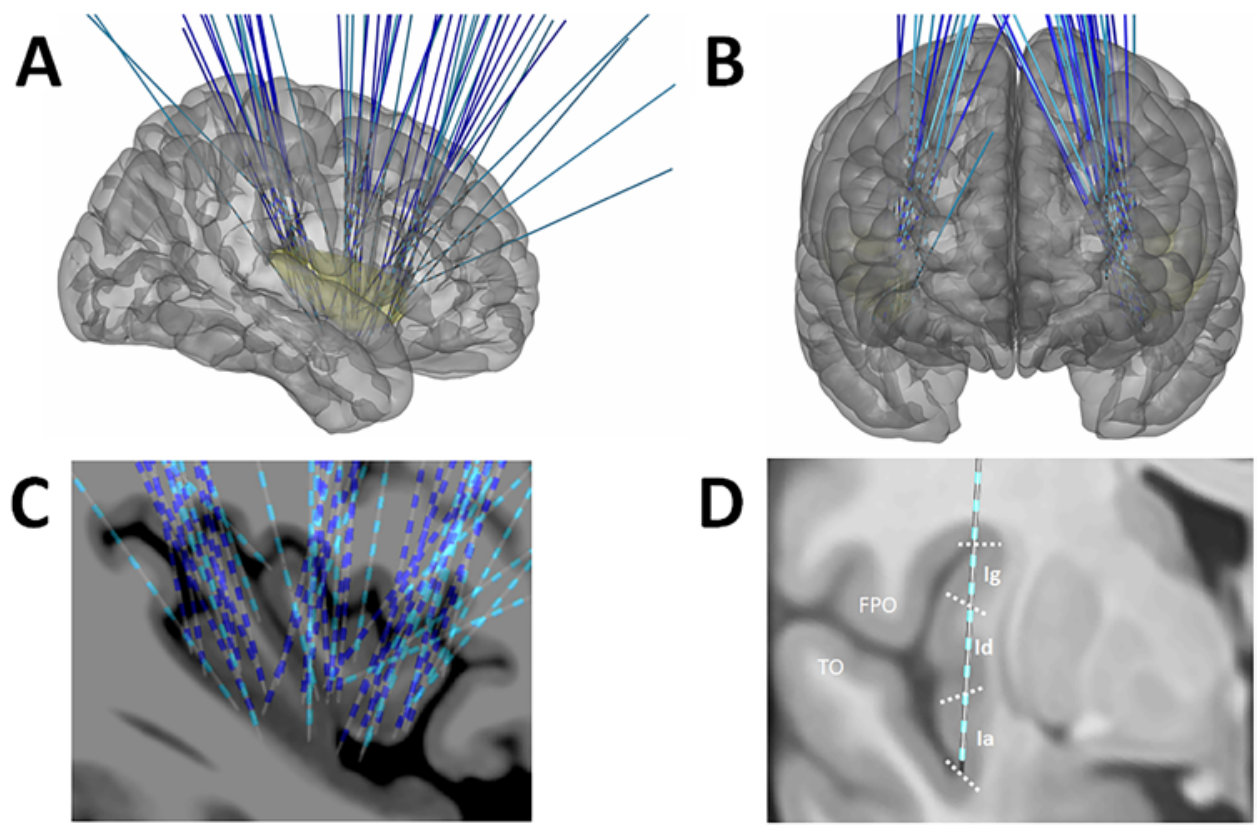

FIG. 1. Insular SEEG trajectory applying an $A O A$ or POA to the insula. Lateral (A) and anterior (B) views of the cortical surface reconstructions demonstrating the applied trajectories (light blue, frame group; dark blue, robot group). Sagittal MR image (C) showing targeting within the insula. The AOA with an anterior frontal entry point at the superior or medial frontal gyrus was applied to target the accessory gyrus as well as the anterior and middle short insular gyri representing social-emotional and cognitive functional networks, respectively. The AOA with a posterior frontal entry point was used to target the region between the middle and posterior short gyri involved in chemosensory circuits. The sensorimotor networks within the anterior and posterior long gyri posterior to the central insular sulcus were targeted by a POA via an entry point at the postcentral gyrus or superior parietal lobule. Coronal MR image (D) showing the tangential course of the SEEG electrodes within the insular cortex covering the ventrodorsal functional circuits as described by Augustine. ${ }^{6} \mathrm{FPO}=$ frontoparietal operculum; la = agranular field; Id = transitional dysgranular field; $\mathrm{lg}$ = granular field; TO = temporal operculum. Figure is available in color online only.

\section{TABLE 2. Summary of implantation procedures}

\begin{tabular}{lccc}
\hline & Overall & Frame Group & Robot Group \\
\hline Mean age, yrs & $25.0 \pm 16.0$ & $23.2 \pm 17.0$ & $26.5 \pm 15.7$ \\
\hline Female/male & $17: 10$ & $9: 3$ & $8: 7$ \\
\hline No. of electrodes & & & \\
$\quad$ Insular & 55 & 28 & 27 \\
Temporal & 67 & 9 & 58 \\
Frontal & 53 & 32 & 21 \\
Parietal & 38 & 22 & 16 \\
Occipital & 7 & 0 & 7 \\
\hline Mean implantation time/ & $11.3 \pm 3.4$ & $15.1 \pm 1.9$ & $9.1 \pm 1.7$ \\
electrode, mins & & & \\
\hline Mean length of & & & \\
trajectories, mm & & & \\
$\quad$ Overall & $42.5 \pm 18.7$ & $39.4 \pm 19.9$ & $44.6 \pm 17.6$ \\
Noninsular & $33.7 \pm 11.1$ & $27.9 \pm 8.6$ & $37.3 \pm 11.0$ \\
Insular & $69.3 \pm 9.5$ & $66.2 \pm 10.6$ & $72.4 \pm 7.1$ \\
\hline Mean EPE, mm & & & \\
$\quad$ Overall & $1.0 \pm 0.7$ & $1.5 \pm 0.6$ & $0.7 \pm 0.5$ \\
Noninsular & $0.9 \pm 0.6$ & $1.5 \pm 0.5$ & $0.7 \pm 0.5$ \\
Insular & $1.1 \pm 0.7$ & $1.6 \pm 0.7$ & $0.7 \pm 0.6$ \\
\hline Mean TPE, mm & & & \\
Overall & $1.6 \pm 0.8$ & $1.5 \pm 0.8$ & $1.6 \pm 0.8$ \\
Noninsular & $1.5 \pm 0.8$ & $1.5 \pm 0.8$ & $1.5 \pm 0.8$ \\
Insular & $1.6 \pm 0.9$ & $1.5 \pm 0.8$ & $1.6 \pm 1.0$ \\
\hline
\end{tabular}

dural electrodes $(n=3)$ were removed from the analysis concerning the surgical procedure time. Insular electrode contacts with epileptogenic onset activity were detected and visualized using MATLAB (MathWorks, Inc.), the SPM 12 toolbox (Penny et al..$^{25}$ ), and the Lead-DBS toolbox (https://www.lead-dbs.org; Fig. 1). The general concept of the Lead-DBS toolbox has been described elsewhere. ${ }^{26}$ In summary, pre- and postoperative MRI scans were coregistered linearly using SPM 12 and then spatial normalized into the MNI_ICBM_2009b_NLIN_ASYM space. ${ }^{27,28}$ Images were corrected for brain shift in postoperative acquisitions by applying a refined affine transform calculated between pre- and postoperative acquisitions that were restricted to a subcortical area of interest as implemented in the brain shift-correction module of the Lead-DBS software. ${ }^{26}$ Finally, SEEG electrodes were manually localized based on postoperative acquisitions using a tool specifically designed for this task. ${ }^{26}$ By this, all SEEG electrodes can be visualized in the MNI ICBM_2009b_NLIN_ASYM space. ${ }^{27}$ Clinical data including seizure origin, secondary epilepsy surgery, and seizure freedom after surgery were documented (Table 2).

\section{Statistical Analysis}

All analyses and statistical tests were performed using MATLAB and IBM SPSS Statistics for Windows (version 26.0, IBM Corp.). Differences in EPE and TPE between noninsular and insular trajectories as well as between the 

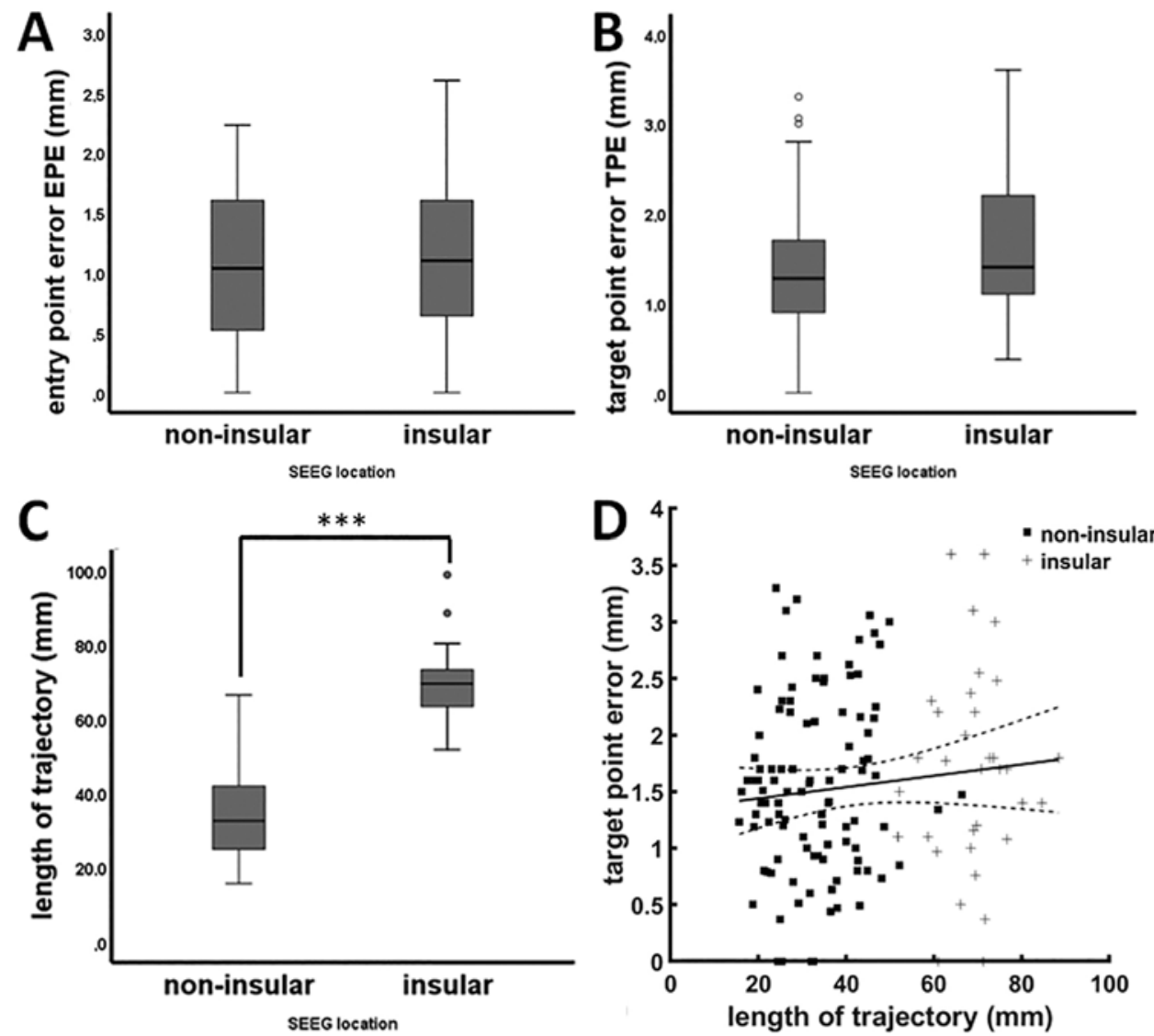

FIG. 2. Boxplots $(A-C)$ and scatterplot (D) showing accuracy of insular and noninsular SEEG electrodes. There were no significant differences in EPE $(\mathbf{A})$ and TPE $(\mathbf{B})$ comparing noninsular and insular electrodes. While the length of noninsular and insular trajectories differed significantly (C), there was no significant correlation between the mean length of the trajectories and the TPE (D).

frame and the robot group were evaluated with an unpaired t-test. Correlation analysis was based on Pearson's correlation. Data are referred to as the mean \pm standard deviation, and boxplots provide additional information about the median and IQR; $\mathrm{p}<0.05$ was considered significant.

\section{Results}

\section{Surgical Procedures and Complications}

A consecutive series of 220 SEEG implantations in 27 patients were included in this retrospective analysis. Ninety-one electrodes were implanted using a framebased procedure in 12 patients, and 129 electrodes were implanted with robot assistance in 15 patients. In total, 55 insular and 165 noninsular electrodes were implanted with a mean overall EPE of $1.0 \pm 0.7$ and a mean overall TPE of $1.6 \pm 0.8$.

Subdural hematoma was observed on postoperative CT in 1 patient, with no neurological deficits $(1 / 27$ patients, $3.7 \% ; 1 / 220$ electrodes, $0.5 \%$ ). The hematoma was evacuated after the condition became chronic (11 days after SEEG implantation and 6 days after finishing SEEG recordings and removal of SEEG electrodes) via two burr holes. The FRE in this case was $0.63 \mathrm{~mm}$, and the mean TPE of the 11 SEEG electrodes was $0.73 \pm 0.39 \mathrm{~mm}$.

\section{Noninsular Versus Insular Trajectories}

The insula was targeted via an AOA or a POA in 55 of the 220 trajectories. When comparing noninsular and insular electrodes, there were no significant differences in EPE (noninsular: $0.9 \pm 0.6 \mathrm{~mm}$ and insular: $1.1 \pm 0.7 \mathrm{~mm}$; $\mathrm{p}=0.358$, unpaired t-test [Fig. 2A]) and TPE (noninsular: $1.5 \pm 0.8$ and insular: $1.6 \pm 0.9 \mathrm{~mm} ; \mathrm{p}=0.876$, unpaired t-test [Fig. 2B]). While the length of noninsular and insular electrodes differed significantly (mean $33.7 \pm 11.1$ and $69.3 \pm 9.5 \mathrm{~mm} ; \mathrm{p}<0.001$, unpaired t-test [Fig. 2C]), there was no significant correlation between the length of the electrodes and the TPE (overall: $r=0.07, p=0.397$; noninsular: $\mathrm{r}=0.03, \mathrm{p}=0.778$ and insular: $\mathrm{r}=0.01, \mathrm{p}=$ 0.930; Pearson's correlation [Fig. 2D]). In contrast, there was a significant positive correlation between EPE and TPE (overall: $r=0.36, p<0.001$; noninsular: $r=0.39, p<$ 0.001 and insular: $r=0.26, p=0.275$; Pearson's correlation). AOA and POA trajectories resulted in a mean of 4.9 \pm 1.4 contacts within the insular cortex.

\section{Frame-Based Versus Robot-Assisted Implantations}

The frame group had a significantly larger EPE when compared with the robot group (frame: $1.5 \pm 0.6$ and robot: $0.7 \pm 0.5 \mathrm{~mm} ; \mathrm{p}<0.001$, unpaired t-test [Fig. 3A]). However, there was no group difference in the TPE (frame: 1.5 

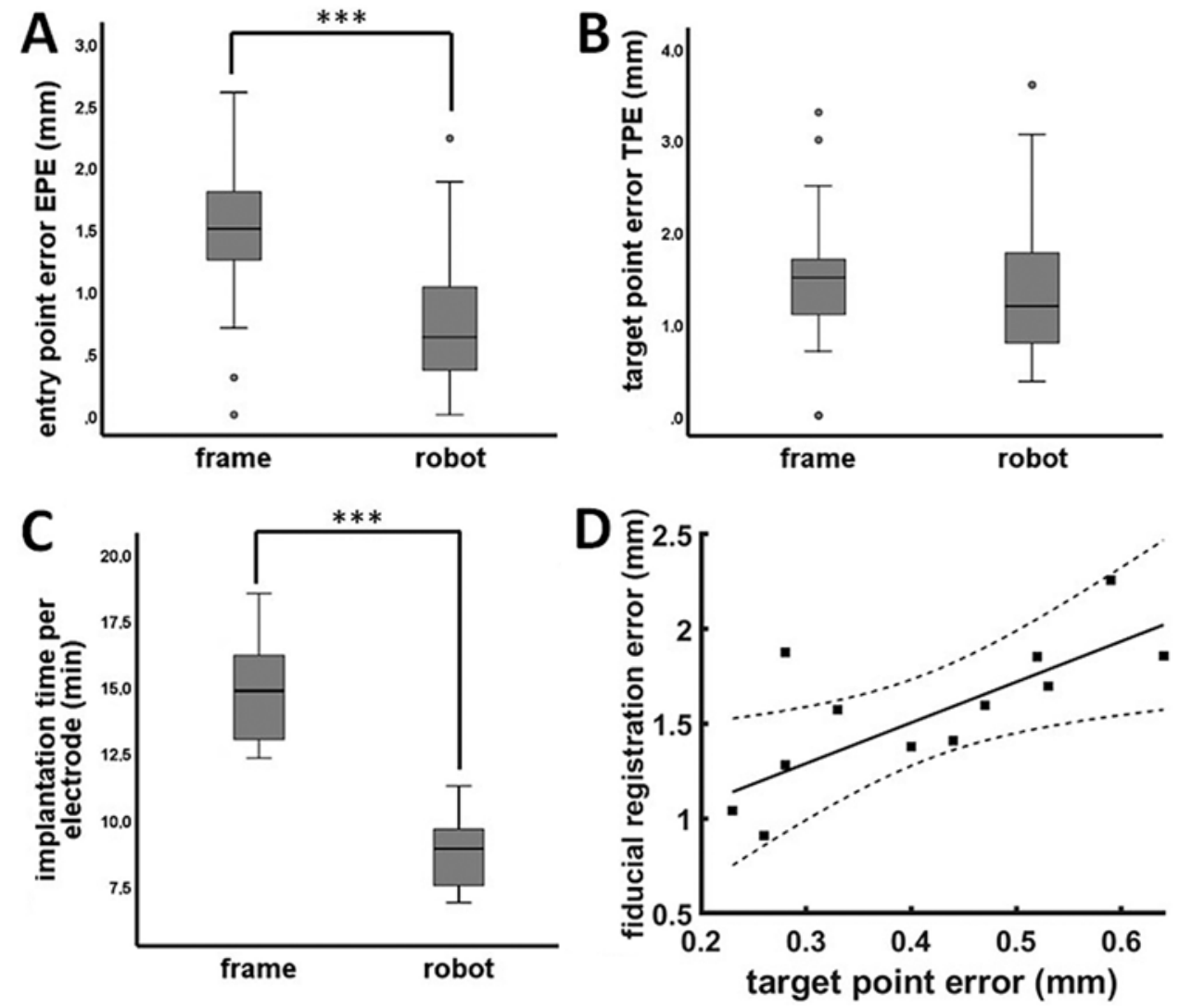

FIG. 3. Boxplots $(A-C)$ and scatterplot (D) showing accuracy of frame-based and robot-assisted SEEG implantations. There was a significantly smaller EPE in the robot group compared with the frame group $(\mathbf{A})$, but there was no group difference in the TPE (B). Notably, robot-assisted surgery necessitated significantly less implantation time per electrode (C). Finally, in robot-assisted surgery, the mean TPE was significantly correlated with the FRE (D).

\pm 0.8 and robot: $1.6 \pm 0.8 \mathrm{~mm} ; \mathrm{p}=0.733$, unpaired t-test [Fig. 3B]). Robot-assisted surgery necessitated significantly less implantation time per electrode (frame: $15.1 \pm 1.9$ and robot: $9.1 \pm 1.7$ minutes; $\mathrm{p}<0.001$, unpaired t-test [Fig. $3 \mathrm{C}])$. For both the frame and the robot group, there was a significant positive correlation between the EPE and the TPE $(r=0.48, p=0.002$ and $r=0.29, p=0.026$, respectively; Pearson's correlation). The electrode length had no impact on the TPE in the frame group $(\mathrm{r}=0.03, \mathrm{p}=0.778$; Pearson's correlation) or in the robot group $(r=0.08, p=$ 0.397; Pearson's correlation).

In the present series of robot-assisted SEEG, intraoperative registration was performed using 5 bone fiducials. The mean registration time was $4.7 \pm 2.1$ minutes, and the mean FRE was $0.4 \pm 0.1 \mathrm{~mm}$. Notably, there was a significant correlation between the FRE and the mean EPE $(\mathrm{r}=0.69, \mathrm{p}=0.042$; Pearson's correlation) as well as the mean TPE $(r=0.73, p=0.007$; Pearson's correlation [Fig. $3 \mathrm{D}])$. Multivariate regression analysis $\left(\mathrm{r}^{2}=0.38, \mathrm{~F}_{(2,102)}=\right.$ $8.65, \mathrm{p}<0.001)$ confirmed that FRE $(\mathrm{B}=2.171,95 \% \mathrm{CI}$ $1109-3232 ; \beta=0.375)$ is the main predictor of TPE in the robot group, in contrast to the electrode length $(\mathrm{B}=0.004$, $95 \% \mathrm{CI}-0.005$ to $0.013 ; \beta=0.081$ ).

\section{Results of the SEEG Recordings}

Epilepsy-related SEEG activity was detected in 66
(25\%) of 266 contacts of 23 (42\%) of 55 electrodes in 15 (56\%) of 27 patients. On an electrode level, the epilepsyrelated activity occurred focally clustered within the anteroposterior and ventrodorsal extent of the insula (Fig. 4). Purely insular seizure onset was detected in only 7 (47\%) of 15 patients; in 6 (40\%) of 15 patients, the ictal SEEG activity was classified as fast propagation. Two (13\%) of 15 patients had multifocal seizure onset zones.

\section{Clinical Outcome of Subsequent Resective Surgery}

Twelve (80\%) of 15 patients with epilepsy-related insular SEEG activity underwent a subsequent surgical procedure, and $5(71 \%)$ of 7 patients with purely insular seizure onset received a lesionectomy of an insular focal cortical dysplasia (FCD). Lesionectomy was suggested in 2 (29\%) of 7 patients; however, resective surgery has not yet been performed. All patients $(5 / 5,100 \%)$ with purely insular seizure onset and an insular lesionectomy had a good outcome (Engel grade I) after the follow-up period. Three $(60 \%)$ of the 5 patients experienced a transient hemiparesis, which completely recovered during follow-up. However, $1(20 \%)$ of the 5 patients had a residual weakness of the contralateral limbs. One $(20 \%)$ of the 5 patients experienced postoperative motoric aphasia, which improved during follow-up. All patients with fast propagation $(\mathrm{n}=$ 6) of SEEG activity to the insular underwent a subsequent 

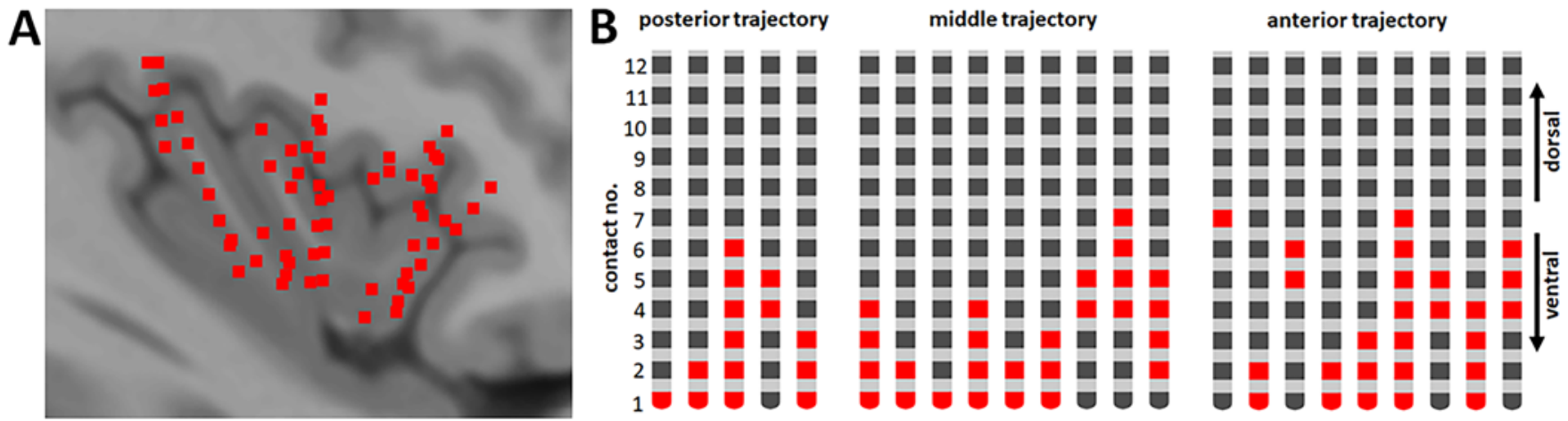

FIG. 4. A: Sagittal MR image overlayed with the electrode contacts (red) depicting epilepsy-related SEEG activity. Epilepsy-related SEEG activity was detected in 66 contacts of 23 electrodes in 15 patients. B: On an electrode level, the epilepsy-related activity occurred focally clustered within the ventrodorsal extent of the insula. However, purely insular seizure onset was detected in 7 of 15 patients. Figure is available in color online only.

resective surgery: lesionectomy of a cortical FCD in 4 $(67 \%)$ or an amygdalohippocampectomy in $2(33 \%)$. All patients with fast propagation of SEEG activity had a good outcome (Engel grade I) after resective surgery. Finally, $2(13 \%)$ of 15 patients had a multifocal seizure onset, 1 $(50 \%)$ of whom was treated with vagus nerve stimulation and had a poor outcome (Engel grade III). The clinical outcome of subsequent resective surgery is summarized in Table 3.

\section{Discussion}

The present study aimed to evaluate the accuracy of insular electrodes placed via AOA and POA during framebased and robot-assisted SEEG implantations. There were no significant differences in EPE and TPE comparing noninsular and insular electrodes, although the length of noninsular and insular trajectories differed significantly. For the frame group, there was no significant correlation between the length of the trajectories and the TPE. Notably, approximately $60 \%$ of the presented electrodes were implanted with robotic assistance. The EPE was significantly smaller in the robot group compared with the frame group. However, there was no group difference in the TPE. Noteworthy, the registration error was the strongest predictor of the TPE in the robot group, although the electrode length had a slight impact on accuracy of electrode placement. Finally, robot-assisted surgery necessitated significantly less implantation time per electrode.

The present study is one of the few studies in which the accuracy of oblique trajectories to the insula has been evaluated. While most of the SEEG trajectories are usually perpendicular to the cortical surface penetrating the brain for a few centimeters, ${ }^{29}$ the insular AOA and POA trajectories necessitate an acute angle and a length of several centimeters (up to $10 \mathrm{~cm}$ in the present study). As the approach angle ${ }^{30}$ and the length of the trajectory are known to affect the target accuracy, insular SEEG electrodes are expected to be susceptible to target errors. However, in the present series, there were no differences in the radial error of insular electrodes in comparison with noninsular electrodes regardless of whether frame-based or robot-assisted implantation was performed. Our accuracy values are in line with those of earlier studies of frame-based and robotassisted SEEG implantations (for a review, see the article by Vakharia et al. ${ }^{31}$ ). Notably, in the present series, the EPE of electrodes placed with robot assistance was significantly smaller than that after frame-based implantation. However, one should consider the fact that the applied stereotactic frame (i.e., CRW, Radionics) is based on the arc-centered principle that tends to maximize surgical precision at the target. In contrast, frameless navigation is known to yield the highest precision at the entry point.$^{32}$ Additionally, the risk of slipping along the skull at the entry point is minimized in robot-assisted surgery by moving the robotic arm as close as possible to the head during drilling. This maneuver is not possible in frame-based implantations. Notably, as most complications in SEEG surgery are associated with the dural and pial puncture, the increased accuracy of the entry point in robot-assisted implantation might help reduce the risk in SEEG implantations. ${ }^{23,33,34} \mathrm{In}$ contrast, there was no difference in target point accuracy

TABLE 3. Outcome of patients with insular epilepsy-related SEEG activity

\begin{tabular}{ccccc}
\hline Epilepsy-Related Insular SEEG Activity $(\mathrm{n}=15)$ & No. of Patients $(\%)$ & 2nd-Stage Surgery & Outcome & Mean FU, mos (range) \\
\hline Purely insular & $7(47.0)$ & Lesionectomy: $5(71.4)^{*}$ & Engel grade I: $5(100)$ & $33.8 \pm 16.3(11.2-48.7)$ \\
\hline Fast propagation & $6(40.0)$ & Lesionectomy: $4(66.7)$ & Engel grade l: $4(100)$ & $24.5 \pm 8.5(16.7-34.5)$ \\
& & AHE: $2(33.3)$ & Engel grade I: (2) & $20.0 \pm 7.1(15.0-25.0)$ \\
\hline Multifocal & $2(13.0)$ & VNS: $1(50.0) ;$ no op: $1(50.0)$ & Engel grade III & 35.6 \\
\hline
\end{tabular}

AHE = amygdalohippocampectomy; $\mathrm{FU}=$ follow-up.

${ }^{*} \mathrm{~A}$ second surgery is pending in 2 of the 7 patients. 
between the robot and frame groups. The reported entry and target point accuracies for robot-assisted SEEG implantation vary over a wide range of values, up to 3 and $4 \mathrm{~mm}$, respectively, ${ }^{17,35}$ strongly depending on the applied registration method. The observed EPE and TPE in this study are comparable to those reported in other studies in which robot assistance and frame-based registration were used. 23,36

Notably, in the present study intraoperative registration was based on bone fiducials. The applied bone fiducials were chosen to exactly fit the size of the robotic pointer (diameter $2 \mathrm{~mm}$ ), thereby reducing the fiducial registration error. Notably, the resulting FRE predicted the EPE and the TPE for the robot-assisted SEEG implantations. This approach allowed an implantation accuracy that was comparable to the frame-based implantations and more precise than robotic procedures with other registration methods, such as laser surface registration. ${ }^{17,23,36}$ However, the use of bone fiducials with a preoperative CT scan (rather than an intraoperative $\mathrm{O}$-arm image) is a unique combination of techniques and might not be applicable to other cohorts. In our opinion, bone fiducial-based registration in robot-assisted SEEG surgery provides a strategy enabling a favorable combination of high accuracy at the level of frame-based registration methods and comparably low discomfort. To our knowledge, this is the first study reporting SEEG accuracies using these bone fiducials. The accuracy of laser surface registration can be affected by a deformation of the skin surface during preoperative imaging or the intraoperative situation as well as by the inaccessibility of the face due to patient positioning. In contrast, surface registration is noninvasive and can reduce radiation exposure for the patient (which is important especially in children). Future developments might provide new solutions for accurate intraoperative registration in robotassisted surgery. ${ }^{37}$

In the present case series, we observed 1 complication, the occurrence of a subdural hematoma after robot-assisted SEEG. This complication was not related to implantation of insular electrodes or registration inaccuracy. The FRE and EPE were comparable to other cases of this series. The subdural bleeding did not result in new neurological deficits and was treated by burr hole evacuation after the condition became chronic (i.e., 6 days after removal of the SEEG electrodes). When considering the high number of trajectories per patient, the risk appears to be small $(1 / 220$ $=0.5 \%)$. However, when the number of patients is considered, the risk greatly increases $(1 / 27=3.7 \%)$. Recent studies have described the risk of symptomatic and asymptomatic bleeding with frequencies of $1 \%$ to $19.1 \% .^{33,38}$

Finally, robot-assisted surgeries necessitated significantly less implantation time per electrode, comparable to previous studies. ${ }^{19,36}$ In a study with 101 robot-assisted SEEG procedures, a mean skin-to-skin time of 130 minutes was reported..$^{19}$ Furthermore, De Benedictis et al. reported a surgery time of 204.3 minutes for the implantation of approximately 11 electrodes. ${ }^{36}$ In contrast, the frame-based implantation doubles the implantation time per electrode due to the necessity for manual application of the coordinates to the stereotactic frame. ${ }^{16,17,39}$ The reduced surgery time might reduce the perioperative risk of thrombosis, postoperative delirium, or infections. Notably, analysis of the implantation time per electrode in this consecutive case series might be biased because all framebased SEEG implantations were performed prior to the installation of the robotic unit, although the procedures were performed by the same surgeon. However, considering the surgeon's learning curve, an additional acceleration of surgical procedure in robot-assisted SEEG implantations is expected..$^{18}$

The OA is historically the most well established as it represents the shortest trajectory to the target ${ }^{11}$ and it facilitates insular and opercular sampling..$^{40}$ In contrast, the OA may not credit the cytoarchitectonic and functional organization in the anteroposterior and the ventrodorsal extension of the insula. ${ }^{11,13}$ Thus, focally clustered epilepsyrelated EEG activity within distinct insular circuits might be missed by orthogonal electrodes. In contrast, the AOA and POA increase the number of serviceable contacts ${ }^{13,14}$ and by the course of the trajectories have the potential for full insular coverage with fewer electrodes. In the present study, the AOA and POA resulted in approximately 5-6 contacts within the insular cortex. This result is in good accordance with the available literature reporting 4-7 contacts per electrode within the insular cortex.12 Similar to other authors, we consider the AOA to be advantageous for covering the anterior insula, whereas the POA provides better electrode coverage of the posterior insula. ${ }^{12,41}$ In fact, the AOA with an anterior frontal entry point at the superior or medial frontal gyrus was applied to target the accessory as well as the anterior and middle short insular gyri representing social-emotional and cognitive functional networks, respectively. ${ }^{5,22}$ The AOA with a posterior frontal entry point was used to target the region between the middle and posterior short gyri involved in chemosensory circuits. ${ }^{5,22}$ The sensorimotor networks within the anterior and posterior long gyri posterior to the central insular sulcus were targeted by a POA via an entry point at the postcentral gyrus or superior parietal lobule. ${ }^{5,22} \mathrm{Ad}-$ ditionally, the trajectories were planned tangential to the insular cortex to cover the ventrodorsal functional circuits as well. ${ }^{4,6,42,43}$ 1) The rostroventral agranular cortex connected to the gyral portion of the anterior cingulate cortex as well as the entorhinal cortex. 2) The intermediate dysganular areas connected to the primary somatosensory cortex, the amygdaloid body, and frontal operculum. 3) The caudodorsal granular part of the insula connected to the primary somatosensory cortex, secondary somatosensory areas, and retroinsular area. In fact, we identified that epilepsy-related SEEG activity showed a clustering in the ventrodorsal direction implying the affection of different functional loops. However, whether these functional loops correlate with the semiology remains unclear and is beyond the focus of this study. ${ }^{7}$ Notably, not every epilepsyrelated SEEG activity indicated seizure onset within the insula. In nearly $50 \%$ of the cases, epilepsy-related SEEG activity was propagated to the insula from another brain area (e.g., frontal FCD or hippocampal sclerosis). Insular SEEG recordings were very sensitive in detecting this difference. Thus, subsequent resective surgery was very efficient in controlling seizures in both purely insular and fast propagation of SEEG activity. As the semiology of insular 
onset seizure might mimic both frontal and temporal origin, ${ }^{7}$ delineating a purely insular onset might help improve the results of resective epilepsy surgery. ${ }^{44}$

\section{Conclusions}

Implantation of insular SEEG electrodes via the AOA or POA is a safe and efficient approach to reach full coverage of the insular anteroposterior and ventrodorsal functional organization. There is no significant difference regarding the SEEG accuracy between insular and noninsular trajectories or between frame-based and robotassisted implantations with bone fiducial registration. The length of the trajectories did not affect the accuracy of the SEEG electrodes significantly. In contrast, TPE was predicted by the entry point accuracy and the registration error during robot-assisted procedures. Robot assistance significantly reduces implantation time without affecting SEEG accuracy even in insular epilepsy.

\section{References}

1. Blount JP, Cormier J, Kim H, et al. Advances in intracranial monitoring. Neurosurg Focus. 2008;25(3):E18.

2. Roberts DW, Jobst BC, Siegel AM, et al. Investigation of extra-temporal epilepsy. Stereotact Funct Neurosurg. 2001; 77(1-4):216-218.

3. Dubeau F, McLachlan RS. Invasive electrographic recording techniques in temporal lobe epilepsy. Can J Neurol Sci. 2000;27(suppl 1):S29-S34.

4. Nieuwenhuys R. The insular cortex: a review. Prog Brain Res. 2012;195:123-163.

5. Kurth F, Eickhoff SB, Schleicher A, et al. Cytoarchitecture and probabilistic maps of the human posterior insular cortex. Cereb Cortex. 2010;20(6):1448-1461.

6. Augustine JR. Circuitry and functional aspects of the insular lobe in primates including humans. Brain Res Brain Res Rev. 1996;22(3):229-244.

7. Jobst BC, Gonzalez-Martinez J, Isnard J, et al. The insula and its epilepsies. Epilepsy Curr. 2019;19(1):11-21.

8. Guenot M, Isnard J, Ryvlin P, et al. Neurophysiological monitoring for epilepsy surgery: the Talairach SEEG method. Indications, results, complications and therapeutic applications in a series of 100 consecutive cases. Stereotact Funct Neurosurg. 2002;77(1-4):29-32.

9. Guenot M, Isnard J, Sindou M. Surgical anatomy of the insula. Adv Tech Stand Neurosurg. 2004;29:265-288.

10. Türe U, Yaşargil DCH, Al-Mefty O, Yaşargil MG. Topographic anatomy of the insular region. J Neurosurg. 1999; 90(4):720-733.

11. Desai A, Bekelis K, Darcey TM, Roberts DW. Surgical techniques for investigating the role of the insula in epilepsy: a review. Neurosurg Focus. 2012;32(3):E6.

12. Afif A, Chabardes S, Minotti L, et al. Safety and usefulness of insular depth electrodes implanted via an oblique approach in patients with epilepsy. Neurosurgery. 2008;62(5)(suppl 2): ONS471-ONS480.

13. Afif A, Minotti L, Benabid A-L, et al. Anatomo-functional organization of the insular cortex in epileptic humans: study using intracerebral electrical stimulation. Neurosurgery. 2008;62(6):1420-1421.

14. Laoprasert P, Ojemann JG, Handler MH. Insular epilepsy surgery. Epilepsia. 2017;58(suppl 1):35-45.

15. Arya R, Mangano FT, Horn PS, et al. Adverse events related to extraoperative invasive EEG monitoring with subdural grid electrodes: a systematic review and meta-analysis. Epilepsia. 2013;54(5):828-839.
16. Guo Z, Leong MCW, Su H, et al. Techniques for stereotactic neurosurgery: beyond the frame, toward the intraoperative magnetic resonance imaging-guided and robot-assisted approaches. World Neurosurg. 2018;116:77-87.

17. Spyrantis A, Cattani A, Woebbecke T, et al. Electrode placement accuracy in robot-assisted epilepsy surgery: a comparison of different referencing techniques including framebased CT versus facial laser scan based on CT or MRI. Epilepsy Behav. 2019;91:38-47.

18. Machetanz K, Grimm F, Schuhmann M, et al. Time efficiency in stereotactic robot-assisted surgery: an appraisal of the surgical procedure and surgeon's learning curve. Stereotact Funct Neurosurg. 2021;99(1):25-33.

19. González-Martínez J, Bulacio J, Thompson S, et al. Technique, results, and complications related to robot-assisted stereoelectroencephalography. Neurosurgery. 2016;78(2): 169-180.

20. Fisher RS, Cross JH, D’Souza C, et al. Instruction manual for the ILAE 2017 operational classification of seizure types. Epilepsia. 2017;58(4):531-542.

21. Engel J, Van Ness PC, Rasmussen TB, Ojemann LM. Outcome with respect to epileptic seizures. In: Engel J, ed. Surgical Treatment of the Epilepsies. 2nd ed. Raven Press; 1993: 609-621.

22. Uddin LQ, Nomi JS, Hébert-Seropian B, et al. Structure and function of the human insula. J Clin Neurophysiol. 2017; 34(4):300-306.

23. Cardinale F, Cossu M, Castana L, et al. Stereoelectroencephalography: surgical methodology, safety, and stereotactic application accuracy in 500 procedures. Neurosurgery. 2013; 72(3):353-366.

24. Fitzpatrick JM. The role of registration in accurate surgical guidance. Proc Inst Mech Eng H. 2010;224(5):607-622.

25. Penny W, Friston K, Ashburner J, et al. Statistical Parametric Mapping: The Analysis of Functional Brain Images. 1st ed. Academic Press; 2007.

26. Horn A, Kühn AA. Lead-DBS: a toolbox for deep brain stimulation electrode localizations and visualizations. Neuroimage. 2015;107:127-135.

27. Avants BB, Epstein CL, Grossman M, Gee JC. Symmetric diffeomorphic image registration with cross-correlation: evaluating automated labeling of elderly and neurodegenerative brain. Med Image Anal. 2008;12(1):26-41.

28. Schönecker T, Kupsch A, Kühn AA, et al. Automated optimization of subcortical cerebral MR imaging-atlas coregistration for improved postoperative electrode localization in deep brain stimulation. AJNR Am J Neuroradiol. 2009;30(10): 1914-1921.

29. Alomar S, Mullin JP, Smithason S, Gonzalez-Martinez J. Indications, technique, and safety profile of insular stereoelectroencephalography electrode implantation in medically intractable epilepsy. J Neurosurg. 2018;128(4):1147-1157.

30. Iordanou JC, Camara D, Ghatan S, Panov F. Approach angle affects accuracy in robotic stereoelectroencephalography lead placement. World Neurosurg. 2019;128:e322-e328.

31. Vakharia VN, Sparks R, O'Keeffe AG, et al. Accuracy of intracranial electrode placement for stereoencephalography: a systematic review and meta-analysis. Epilepsia. 2017;58(6): 921-932.

32. Zrinzo L. Pitfalls in precision stereotactic surgery. Surg Neurol Int. 2012;3(2)(suppl 1):S53-S61.

33. McGovern RA, Ruggieri P, Bulacio J, et al. Risk analysis of hemorrhage in stereo-electroencephalography procedures. Epilepsia. 2019;60(3):571-580.

34. McGovern RA, Butler RS, Bena J, Gonzalez-Martinez J. Incorporating new technology into a surgical technique: the learning curve of a single surgeon's stereo-electroencephalography experience. Neurosurgery. 2020;86(3): E281-E289. 
35. Brandmeir NJ, Savaliya S, Rohatgi P, Sather M. The comparative accuracy of the ROSA stereotactic robot across a wide range of clinical applications and registration techniques. $J$ Robot Surg. 2018;12(1):157-163.

36. De Benedictis A, Trezza A, Carai A, et al. Robot-assisted procedures in pediatric neurosurgery. Neurosurg Focus. 2017;42(5):E7.

37. Cardinale F, Rizzi M, d'Orio P, et al. A new tool for touchfree patient registration for robot-assisted intracranial surgery: application accuracy from a phantom study and a retrospective surgical series. Neurosurg Focus. 2017;42(5):E8.

38. De Barros A, Zaldivar-Jolissaint JF, Hoffmann D, et al. Indications, techniques, and outcomes of robot-assisted insular stereo-electro-encephalography: a review. Front Neurol. 2020;11:1033.

39. Roessler K, Sommer B, Merkel A, et al. A frameless stereotactic implantation technique for depth electrodes in refractory epilepsy using intraoperative magnetic resonance imaging. World Neurosurg. 2016;94:206-210.

40. Aydin Ü, Vorwerk J, Dümpelmann M, et al. Combined EEG/ MEG can outperform single modality EEG or MEG source reconstruction in presurgical epilepsy diagnosis. PLoS One. 2015;10(3):e0118753.

41. Surbeck W, Bouthillier A, Weil AG, et al. The combination of subdural and depth electrodes for intracranial EEG investigation of suspected insular (perisylvian) epilepsy. Epilepsia. 2011;52(3):458-466.

42. Stefan H, Buchfelder M. Diagnosis of sylvian and perisylvian epilepsies. Excitation symptoms of the insula reilii. Article in German. Nervenarzt. 2007;78(10):1175-1181.

43. Menon V, Gallardo G, Pinsk MA, et al. Microstructural organization of human insula is linked to its macrofunctional circuitry and predicts cognitive control. eLife. 2020;9:e53470.
44. Isnard J, Guénot M, Ostrowsky K, et al. The role of the insular cortex in temporal lobe epilepsy. Ann Neurol. 2000;48(4): $614-623$.

\section{Disclosures}

The authors report no conflict of interest concerning the materials or methods used in this study or the findings specified in this paper.

\section{Author Contributions}

Conception and design: Naros, Machetanz. Acquisition of data: Naros, Machetanz. Analysis and interpretation of data: Naros, Machetanz. Drafting the article: Naros, Machetanz. Critically revising the article: Naros, Grimm, Wuttke, Kegele, Lerche, Tatagiba, Rona, Gharabaghi, Honegger. Reviewed submitted version of manuscript: Naros. Approved the final version of the manuscript on behalf of all authors: Machetanz. Statistical analysis: Naros, Grimm, Wuttke. Administrative/technical/ material support: Kegele, Lerche, Tatagiba, Rona, Gharabaghi, Honegger. Study supervision: Naros.

\section{Correspondence}

Georgios Naros: Eberhardt Karls University, Tuebingen, Germany. georgios.naros@med.uni-tuebingen.de. 